\title{
DINÂMICAS ALIMENTARES ALTERNATIVAS E A BUSCA DE UMA GASTRONOMIA SUSTENTÁVEL: CONSUMO DE PRODUTOS LOCAIS EM RESTAURANTES DE BRASÍLIA
}

\section{ALTERNATIVE FOOD DYNAMICS AND THE SEARCH FOR A SUSTAINABLE GASTRONOMY: LOCAL PRODUCTS CONSUMPTION IN BRASILIA RESTAURANTS}

\author{
Jessica P. Garcia1; Tainá B. Zaneti²; Janaína D.A.S. Diniz ${ }^{3}$; Stéphane Guéneau ${ }^{4}$ \\ ${ }^{1}$ Universidade de Brasília. jessicapg15@gmail.com \\ ${ }^{2}$ Universidade de Brasília. tainazaneti@gmail.com \\ ${ }^{3}$ Universidade de Brasília. janadinizbr@gmail.com \\ ${ }^{4}$ CIRAD. stephane.gueneau@cirad.fr
}

\author{
Grupo de Trabalho (GT): 11 - Abastecimento, segurança alimentar e nutricional e \\ dinâmicas de consumo
}

\begin{abstract}
Resumo
Nestes últimos anos, as críticas ao sistema agroalimentar dominante no Brasil levaram à emergência de sistemas alimentares alternativos. Nestes sistemas, o papel dos atores gastronômicos começa a ter interesse na literatura brasileira. Contudo, os efeitos das práticas e inovações implementadas por esses atores gastronômicos nas mudanças nos sistemas alimentares, particularmente nos contextos urbanos, foram pouco estudados.

Este artigo busca compreender como as relações existentes entre os restaurantes de Brasília e produtores locais têm contribuído para a consolidação de sistemas alimentares urbanos alternativos. Para isso foi realizada uma pesquisa qualitativa, com entrevistas semiestruturadas e uma oficina participativa com os atores ligados aos restaurantes que atendiam a pelo menos um dentre dois critérios de seleção: uso de ingredientes da sociobiodiversidade do Cerrado e/ou de agricultores familiares locais no seu cardápio, e participação em projetos ambientais.

Os principais apontamentos dessa contribuição estão relacionados à percepção de novas iniciativas desencadeadas por esses restaurantes, com a agregação de outros serviços como educação alimentar, formação (oficinas), funcionamento como pontos de encontro de coprodutores de comunidades que sustentam a agricultura (CSA), ou como pontos de venda de produtos da agricultura familiar e da sociobiodiversidade, o que contribui para $o$ estabelecimento de sistemas alimentares mais sustentáveis.
\end{abstract}

Palavras-chave: gastronomia sustentável; sistemas alimentares alternativos; circuitos curtos; produtos locais. 


\section{Abstract}

In recent years, criticisms of the dominant agri-food system in Brazil led to the emergence of alternative food systems. In these systems, the role of gastronomic actors begins to have interest in Brazilian literature. However, the effect of practices and innovations implemented by these gastronomic actors in changes in food systems, particularly in urban contexts, has been little studied.

This article seeks to understand how existing relations between the Brasilia restaurants and local producers have contributed to the consolidation of alternative urban food systems. For this a qualitative research was carried out, with semi-structured interviews and a participatory workshop with the actors linked to the restaurants that met at least one of two selection criteria: use of socio-biodiversity ingredients of the Cerrado and/or from local family farmers in their menu, and participation in environmental projects.

The main notes of this contribution are related to the perception of new initiatives launched by these restaurants, with the aggregation of other services such as food education, training (workshops), functioning as a meeting point of co-producers of community supported agriculture (CSA), or as selling points of products of family farming and socio-biodiversity, contributing to the establishment of more sustainable food systems.

Keywords: sustainable gastronomy; sustainable food systems; short circuits; local products.

\section{Introdução}

Cada vez mais o modelo hegemônico de produção, processamento e distribuição de alimentos tem sido questionado, por causar inúmeros conflitos que abrangem questões sociais, ambientais e de saúde, observadas na atual sindemia global, conjunto de pandemias de obesidade, desnutrição e mudanças climáticas (CARVALHO, 2021), mas também pelos limites ou exclusão de pequenos produtores no que se refere ao acesso a mercados.

A partir da crítica das contradições dos sistemas agroalimentares dominantes, tem sido possível identificar alternativas viáveis por meio do fortalecimento de redes alimentares alternativas e do comércio justo (GOODMAN, DUPUIS, GOODMAN, 2012). Dessa forma, os sistemas agroalimentares alternativos estão em busca de novas relações entre o campo e a cidade, visando uma relação mais próxima e justa entre quem produz e quem consome, podendo ser estudados por meio das estruturas dos canais curtos de comercialização (DAROLT et al., 2016).

Já o campo da gastronomia na contemporaneidade tem se mostrado como um local de inovação para práticas de sustentabilidade no sistema alimentar (PEREIRA et al., 2019). Neste cenário, é crescente o número de chefs, em todo o mundo, que escolhem ingredientes notados de características sustentáveis, por serem produzidos de forma orgânica, artesanal e por serem típicos ou nativos da biodiversidade de suas regiões (ZANETI, 2017; PEREIRA et al., 2019; ARAUJO; FINOCCHIO, 2020). No Brasil e em países da América Latina, como Peru e México, e na África do Sul, a cozinha tem se mostrado um espaço de inserção de espécies nativas que são pouco utilizadas e/ou não possuem uma cadeia produtiva/de valor estruturada, mas com grande potencial desses alimentos para (re)integrar a cultura alimentar local. Essas ações são lideradas por chefs de cozinha que, preocupados com as questões socioambientais locais, promovem a valorização da alimentação regional com uso e divulgação de alimentos preparados a partir de plantas nativas, aproximação e fortalecimento da agricultura familiar e da relação campo e cidade e do reaproveitamento de alimentos (ZANETI, 2017; PEREIRA et al., 2019; NIEDERLE; SCHUBERT, 2020). 
No caso de produtos do bioma Cerrado, alguns autores buscam entender a gastronomia como meio de valorização dos produtos regionais através do processo de construção de mercados para estes produtos (ZANETI; BALESTRO, 2015; GUÉNEAU et al., 2017; DUARTE et al., 2020, 2021). Já se sabe também que o incentivo ao uso de produtos da sociobiodiversidade pode ajudar na conservação do Cerrado, pois o agroextrativismo no Cerrado está associado à conservação e manutenção do bioma (GUÉNEAU et al., 2020; NOGUEIRA; FLEISCHER, 2005). Apesar disso, ainda é necessário compreender melhor a relação entre a gastronomia, o consumo desses produtos e os impactos, tanto positivos como negativos, gerados nessas relações.

Sabendo que já existem iniciativas privadas e políticas públicas para promover a (re)valorização de produtos nativos do cerrado, mas também produtos orgânicos e agroecológicos, nos sistemas alimentares da cidade de Brasília (GUÉNEAU et al., 2021), buscamos responder ao questionamento: como a dinâmica alimentar urbana em Brasília tem se estruturado para contribuir com os sistemas agroalimentares alternativos no bioma Cerrado? Para responder a essa questão a pesquisa analisou as relações de atores-chave dos restaurantes inseridos nos sistemas alimentares alternativos de Brasília que estão fazendo uso de produtos da sociobiodiversidade e envolvidos em outras práticas ligadas à sustentabilidade, na busca por uma gastronomia sustentável. Dessa forma, o objetivo deste artigo é discutir como as relações atualmente existentes entre restaurantes de Brasília e produtores locais têm contribuído para a consolidação de sistemas alimentares alternativos no Cerrado.

O texto está dividido em quatro seções, além desta introdução, iniciando com uma breve abordagem teórica sobre sistemas alimentares alternativos e gastronomia contemporânea. Na seção 3 apresentamos a metodologia de pesquisa usada para a coleta e análise dos dados, seguido da apresentação e discussão dos dados levantados na pesquisa de campo e, no último tópico, das considerações finais a respeito da pesquisa apresentada.

\section{Conceitos e contexto da pesquisa}

Os sistemas alimentares alternativos podem ser compreendidos como o arranjo de práticas que apontam possibilidades para resolver os limites e contradições do modelo agroalimentar hegemônico (DEVERRE; LAMINE, 2010). Essas contradições são percebidas pela crise alimentar atual através da massiva homogeneização do padrão alimentar, somada à invisibilização da produção, que gera impactos na saúde humana, como, por exemplo o aumento significativo dos casos de obesidade, e uma profunda desconexão do ser humano com a natureza (SCHNEIDER; CRUZ; MATTE, 2016). Essas práticas dos sistemas alimentares alternativos são estudadas principalmente através de experiências que envolvem temas como comércio justo, canais curtos de comercialização, valorização da produção local, alimentos diferenciados, selos e qualidades, articulando a oferta dos produtores a "códigos" de produção específicos (RENTING; MARSDEN;BANKS, 2017).

Os canais de comercialização são as alternativas possíveis para a disponibilização de um produto para consumidores finais (TELLES; STREHLAU, 2006) e que, em estruturas formais ou informais, conseguem atender aos interesses tanto de produtores como de consumidores, mesmo quando os produtos passam por vários níveis de intermediações. Quando se tem canais ou circuitos curtos, as intermediações são mínimas ou inexistentes. De acordo com Darolt (2011), para ser considerado circuito curto, a comercialização deve ser feita por venda direta do produtor ao consumidor ou por meio de um único intermediário. No primeiro grupo o autor menciona a entrega de cestas em domicílio, venda direta dentro ou fora da propriedade, feiras livres, vendas para programas governamentais, enquanto que no segundo tem-se, por exemplo, lojas virtuais, lojas especializadas, restaurantes, pequenos mercados, entre outros. Gazolla (2011) identificou a presença de circuitos curtos de comercialização em cinco 
diferentes tipos de mercado em pesquisa envolvendo agroindústrias familiares no Sul do Brasil: institucional, de venda direta, de eventos, pontos de venda formais e de organizações sociais em rede.

No caso de produtos da biodiversidade nativa ou da sociobiodiversidade do Cerrado, e também com origem na produção familiar, alguns circuitos alternativos vêm conseguindo modificar as relações de dependência dos produtores agroextrativistas a circuitos longos, conhecidos por possuírem historicamente muitos intermediários (DINIZ; CERDAN, 2017). O agroextrativismo combina a coleta de recursos da biodiversidade nativa com o cultivo e a criação de animais (NOGUEIRA; FLEISCHER, 2005). Em diversas regiões do Cerrado esse sistema de produção específico tem contribuído para a manutenção de diversas famílias no campo pelo incremento de renda possibilitado nos últimos anos pela inserção de produtores agroextrativistas em circuitos de comercialização de produtos da biodiversidade do Cerrado tem (BISPO et al., 2020).

A esfera da gastronomia começa a incorporar algumas práticas que são relacionadas à alimentação contemporânea. Essas práticas da alimentação contemporânea abarcam aspectos da sustentabilidade, a exemplo dos movimentos que visam a redução do consumo de carne e a redução da produção de monoculturas, como também, um movimento de cadeias curtas de alimentos, privilegiando alimentos comprados diretamente do agricultor de base orgânica ou agroecológica (PORTILHO; CASTAÑEDA; CASTRO, 2011).

Com o envolvimento da gastronomia com a alimentação contemporânea, alguns autores, como Steinberger (2010), Barber (2015) e Niederle e Schubert (2020), argumentam que os chefs assumem um papel social e político em relação à alimentação - o ativismo gastronômico - no sentido de, ao usarem tais alimentos em suas cozinhas e justificarem essas escolhas, passam a exercer influência e a educar seus consumidores em direção a uma gastronomia sustentável.

O conceito de gastronomia sustentável ainda é um tema em debate e, segundo Krause e Bahls (2013), apesar de ainda não haver uma definição para este conceito, é possível elencar alguns aspectos que podem tornar a gastronomia uma atividade sustentável. Entre elas, destacam-se a elaboração de cardápios sustentáveis, baseados na sazonalidade, na produção orgânica e local, aspectos culturais, rotatividade; a escolha dos ingredientes, baseada na embalagem e na estocagem; e educação ambiental, com ensino e treinamento da mão-de-obra do setor sobre este assunto. Esses aspectos também foram sintetizados por Zanella (2020) ao elencar dez princípios para uma gastronomia sustentável.

O contexto de Brasília, como capital federal e no centro do Cerrado, é emblemático, uma vez que o bioma sofre com a expansão do agronegócio, com altas taxas de desmatamento e diversos danos socioambientais (ELOY et al., 2018). Brasília também se destaca atualmente como uma cidade jovem (60 anos em 2020) que está desenvolvendo sua identidade gastronômica e já recebe destaque como $3^{\circ}$ maior polo gastronômico do Brasil (Congresso em Foco, 2017). A cidade é um local que concentra o poder público, por ser a capital do país, e que também possui uma população dotada de elevado capital financeiro e intelectual, favorecendo a preocupação de importante parte da população com temas relacionados à sustentabilidade.

\section{Metodologia}

A metodologia da pesquisa foi qualitativa, realizada em duas etapas. A primeira etapa contou com entrevistas com atores-chave e a segunda consistiu na realização de um evento participativo para estimular o diálogo entre os atores, buscando atender à metodologia do 
projeto URBAL ${ }^{1}$ - Inovações urbanas para sistemas alimentares sustentáveis (VALETTE et al., 2019). Foram conduzidas 26 entrevistas semiestruturadas entre 2018 e 2019, sendo 16 entrevistas com chefs de cozinha e proprietários de restaurantes de Brasília, 1 entrevista com cooperativa, 6 entrevistas com produtores (Comunidade que Sustenta a Agricultura-CSA e Agroextrativista), 2 entrevistas com intermediários comerciais, e 1 com responsável por programa gastronômico que atua em parceria com a Secretaria de Educação do Distrito Federal. A partir da transcrição dessas entrevistas, utilizou-se da técnica de análise de conteúdo como ferramenta de análise dos dados.

A escolha dos estabelecimentos se deu por meio da definição de alguns critérios, previamente estabelecidos com base na literatura de sistemas alimentares alternativos e sustentabilidade na gastronomia (CASSOL; SCHNEIDER, 2015; DAROLT et al., 2016; ZANETI; SCHNEIDER, 2017). Esses critérios foram: (1) uso de ingredientes da flora local; e/ou (2) uso de produtos da agricultura familiar da região, e/ou (3) participação em projetos ambientais que envolvessem a destinação adequada de resíduos e/ou aproveitamento integral de alimentos. A busca por estes chefs ocorreu a partir da técnica bola de neve, enquanto a quantidade de chefs selecionados e entrevistados atendeu ao princípio da saturação.

A segunda etapa da pesquisa se desenvolveu por meio de uma oficina participativa organizada em Brasília em junho de 2019. Essa oficina reuniu vários atores de interesse da pesquisa. A oficina permitiu analisar as inovações e ações implementadas que podem contribuir na transformação do sistema alimentar na direção de um sistema mais sustentável, utilizando uma metodologia na configuração de um grupo focal. Participaram dessa oficina: chefs de cozinha, agricultores familiares, povos tradicionais, representantes de cooperativas da cadeia produtiva de produtos do Cerrado, representantes do poder público e pesquisadores. A oficina participativa foi realizada em duas etapas e foi conduzida de modo a permitir o amplo diálogo entre os atores, com perguntas-guia pré-estabelecidas pelos pesquisadores. Em cada rodada de perguntas os atores eram convidados a falar sobre o assunto e a interagir com os demais presentes. Para contribuir na análise, a oficina teve registro de imagem e som para a produção de um vídeo resumo, e a gravação de áudio foi transcrita para análise de conteúdo.

\section{Resultados e discussão}

A partir das entrevistas e reflexões sobre o seminário temático realizado no âmbito desta pesquisa, foi possível perceber a vasta quantidade de relações/conexões que já existem nesse sistema que orbita a gastronomia sustentável em Brasília. Chamamos de teia da sustentabilidade o conjunto dessas ligações, apresentadas na figura 1, e classificamos esses atores em cinco categorias: intermediários, produtores, consumidores, poder público, e restaurantes.

\footnotetext{
${ }^{1}$ Projeto internacional de pesquisa sobre inovações urbanas em oito cidades de diferentes continentes, que visa desenvolver e testar metodologia para mapear os caminhos de impacto que partem das inovações urbanas para todas as dimensões da sustentabilidade dos sistemas alimentares. Para mais informações, acessar: https://www.urbalfood.org/the-project/.
} 


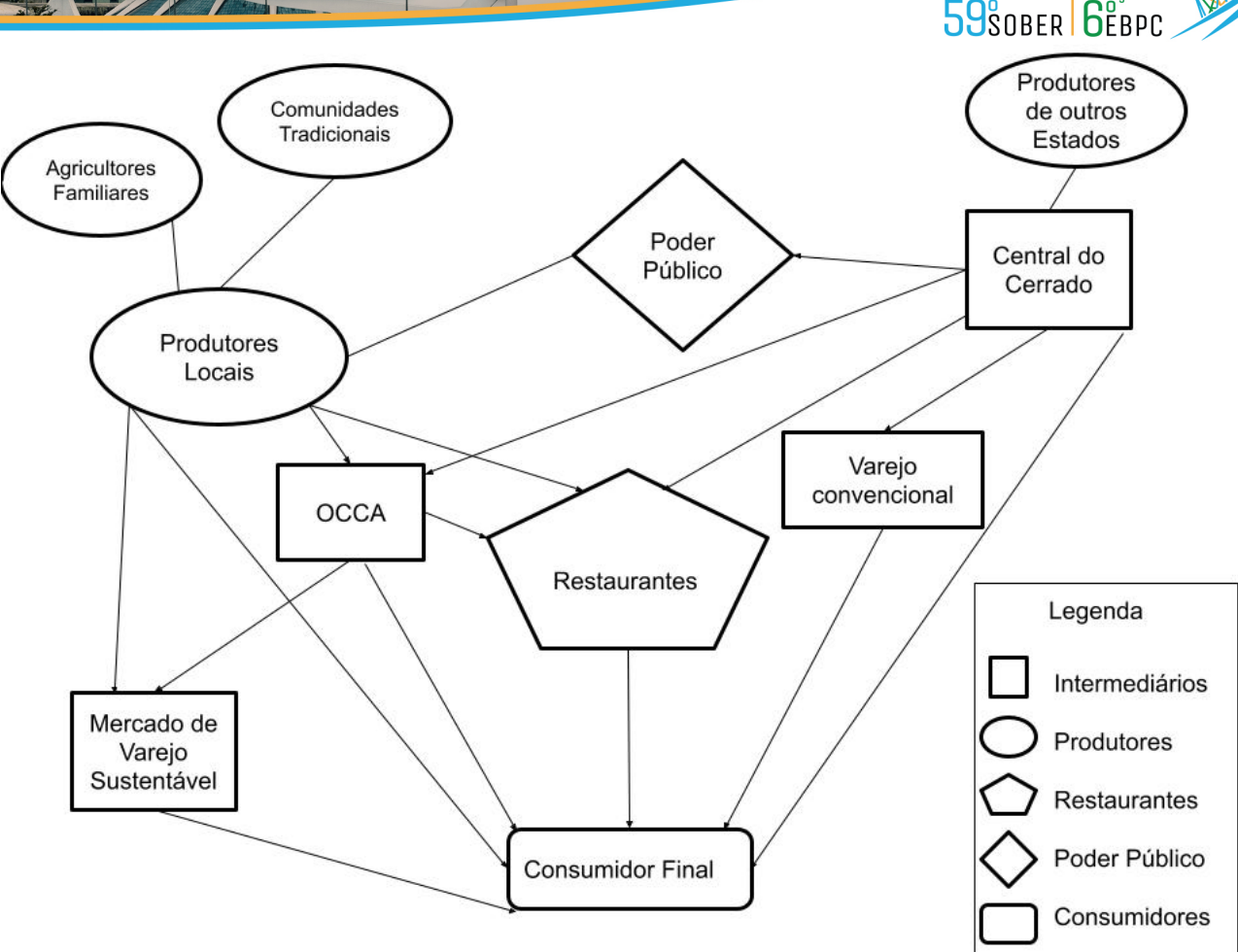

Figura 1: teia da sustentabilidade. Fonte: elaboração dos autores

A categoria de intermediários refere-se às cooperativas, associações e mercados que possuem o diferencial de proximidade com os produtores e comercializam os produtos dos agroextrativistas e/ou agricultores abordados nesta pesquisa. A proximidade com os produtores permite que esses intermediários não quebrem as relações entre produtores e consumidores, conseguindo assim repassar informações que atribuem valores específicos aos produtos, informações adequadas sobre a produção e produtor, transmitindo, assim, confiança nesse processo. Esse tipo de intermediação é citado na literatura que estuda reciprocidade como uma corretagem (SABOURIN, 2013). Os intermediários entrevistados foram a Cooperativa Central do Cerrado, a rede de comercialização OCCA - Organização Coletiva do Cerrado Agroecológico, e o mercado Sustentável EVOLUA.

A Central do Cerrado é uma cooperativa de segunda ordem, fundada em 2004, que tem como objetivo reunir diversas organizações comunitárias que desenvolvem atividades produtivas a partir do uso sustentável da biodiversidade dos biomas Cerrado e Caatinga, com intenção de divulgar e inserir produtos comunitários de uso sustentável em mercados regionais, nacionais e internacionais. A Organização Coletiva do Cerrado Agroecológico (OCCA) é uma rede de comercialização de produtos locais, de comunidades tradicionais e agroindústrias familiares que visa agregar valor a esses produtos, possibilitar uma maior diversidade de oferta aos clientes e consumidores dos produtos da sociobiodiversidade, que também possui uma CSA onde inserem, além da própria produção agroecológica, os produtos do Cerrado. O mercado EVOLUA surge com a proposta de ser um mercado com lixo zero, com nenhuma embalagem de plástico de uso único, e que prioriza a venda de produtos locais. O espaço também proporciona eventos como palestras e debates sobre temas ambientais e funciona como ponto de convivência para CSAs.

Os produtores representam um conjunto diverso entre agricultores familiares, agroextrativistas e comunidades tradicionais, que possuem vínculo de comercialização em alguns estabelecimentos de Brasília. Cada produtor possui sua diversidade de canais de comercialização para escoar sua produção e a venda vinculada ao setor da gastronomia 
representa um dentre esses canais. Alguns conseguem estabelecer um contato direto com os chefs/donos. Esse contato direto foi relatado como meio preferencial por alguns chefs, pois desse modo é possível uma maior negociação de preço, por exemplo. Através dos intermediários os chefs conseguem acessar também a produção de outros estados, assim como uma diversidade e volume maior de produção.

Na teia da sustentabilidade entram como poder público os órgãos do governo distrital relacionados à assistência técnica - EMATER-DF, e os setores que trabalham com a agricultura familiar do Distrito Federal, como a SEAGRI - Secretaria de Agricultura. Em alguns setores desses órgãos existem pessoas-chave que defendem um trabalho vinculado à promoção e fortalecimento da agroecologia, formando assim parcerias importantes nesse setor.

Consumidores são o público final que tem a possibilidade de consumir os produtos da sociobiodiversidade tanto nos restaurantes quanto comprando com os produtores ou intermediários. Esses consumidores podem vincular o consumo desses produtos a diversos valores de consumo, desde um consumo consciente por buscar uma valorização do bioma local, quanto ao fato de representarem uma questão de status ou um produto gourmet (GARCIA et al., 2020)

Os restaurantes representam tanto os chefs de cozinha quanto os donos de estabelecimentos entrevistados. Esses chefs aderem à rotina dos empreendimentos algumas práticas sustentáveis que são resumidas em quatro características principais. Na ordem de maior frequência dessas práticas destacam-se a composição do cardápio dos estabelecimentos ser constituída por alimentos advindos de produtores locais; o uso de produtos orgânicos; o uso de frutos do Cerrado na elaboração dos pratos; e a preocupação com os resíduos gerados. A partir dessas práticas os dois empreendimentos que tiveram maior destaque foram o restaurante Buriti Zen e o projeto gastronômico Mesa pra Doze.

Nas sub-seções seguintes abordaremos primeiramente esses dois casos destacados e, na sequência, outros aspectos presentes entre os demais restaurantes de Brasília, que possibilitam ou dificultam suas contribuições aos sistemas alimentares alternativos locais.

\subsection{Buriti Zen e Mesa pra Doze: experiências de uma gastronomia em busca da sustentabilidade}

$\mathrm{O}$ restaurante Buriti Zen pertence à chef de cozinha Ana Paula Boquadi. O empreendimento surgiu a partir do histórico de estudos e envolvimento pessoal de sua proprietária com a temática de alimentação vegana entre os anos de 2009 e 2014. Após esse período o Buriti Zen iniciou com a produção e venda de marmitas veganas que eram comercializadas através da entrega via motoboys, porém essa configuração não permitia o contato direto com seus consumidores. Essa necessidade de aproximação era percebida tanto pelos clientes, que cobravam um local para a convivência quanto pela sua proprietária, que via importância nessa proximidade. Esse contexto motivou a abertura de um restaurante em um bairro próximo ao centro de Brasília, local mais acessível ao público-alvo, formado geralmente por pessoas que já possuem uma preocupação socioambiental e que buscam restaurantes que demonstrem práticas de sustentabilidade.

Essas práticas se tornaram um grande diferencial do Buriti Zen, pois a prioridade do estabelecimento era fornecer pratos veganos, orgânicos com produtos da sociobiodiversidade local e sem a presença de alimentos provenientes de grandes monoculturas e commodities, como a soja. Na prática isso foi possível através do contato com os produtores locais e comunidades tradicionais que são os principais fornecedores do restaurante, e da adequação do cardápio que respeita a sazonalidade das espécies vegetais e que insere também os produtos da sociobiodiversidade do Cerrado como ingredientes principais e rotineiros no cardápio. Além disso o restaurante ainda atua como um local de valorização das comunidades tradicionais e 
produtores locais, pois divulga e promove o contato dos consumidores com os produtores através da comercialização dos produtos das comunidades tradicionais, da abertura do restaurante como ponto de convivência para grupos de consumo de CSA e da elaboração de cursos sobre a culinária tradicional com a participação de detentores de saberes tradicionais.

Compartilhando de vários desses princípios, o projeto gastronômico Mesa para Doze foi idealizado por Mateus Zanella, pesquisador da temática de agricultura sustentável e chef de cozinha com foco em alimentação sustentável. A partir desses trabalhos e pesquisas desenvolvidos em alguns países Europeus e principalmente após sua experiência em Berlim, Alemanha, propôs princípios necessários para uma gastronomia sustentável. Dentre os princípios, há o foco no desperdício zero dos alimentos; uso de plantas alimentícias não convencionais - PANCs; uso de produtos agroecológicos; respeito à sazonalidade; criação de elos do "produtor à mesa", priorizando também a compra direta do produto; exploração do uso de produtos da sociobiodiversidade; e busca por uma dieta sustentável, priorizando os vegetais. Ao retornar ao Brasil, Zanella criou o Mesa pra Doze, para colocar em prática esses princípios com a proposta de servir até 12 pessoas semanalmente durante dois dias. Para ampliar a relação entre produção e consumo, antes de servir os pratos, era feita uma explicação sobre quais ingredientes estavam presentes e também sobre suas origens, numa tentativa de seguir o princípio de relação do "produtor à mesa".

A viabilidade desses dois empreendimentos pode ser compreendida primeiramente pela atitude ativista de seus idealizadores, de priorizarem favorecer a produção local, levando em consideração também a valorização dos produtos da sociobiodiversidade e por seu públicoalvo já buscar estabelecimentos com fatores de sustentabilidade.

\subsection{Oportunidades e obstáculos à participação dos restaurantes de Brasília em sistemas alimentares alternativos}

\section{Confluência entre sistemas orbitais independentes}

A gastronomia por si só pode ser considerada um sistema com suas especificidades. Quando a gastronomia contemporânea traz para perto de seu sistema elementos da preocupação socioambiental, ela passa a se conectar com outros sistemas que também já possuem suas lógicas de funcionamento, como no caso dos mercados da sociobiodiversidade. Desse modo a comercialização de produtos da sociobiodiversidade não depende da gastronomia, mas, com a parceria e divulgação na ideia de valorização dos produtos e produtores, pode existir uma relação "ganha-ganha".

O aumento do interesse de chefs de cozinha de Brasília por ingredientes únicos e da biodiversidade local mostra essa abertura de novas oportunidades e conexões. Alguns ingredientes, como o cajuzinho (Anacardium humile) e a baunilha do Cerrado (Vanila edwalli) se mostraram com um potencial aumento de renda para os agroextrativistas das comunidades tradicionais. Nas entrevistas e na oficina participativa foi relatado que após o Buriti Zen começar a comprar cajuzinho, outros chefs e restaurantes passaram a procurar também os mesmos agroextrativistas para outras compras. Essa circulação de contatos e compras pontuais gera oportunidade de aumento de renda para os agricultores, porém ainda numa relação instável por não haver frequência determinada. Para além dessas vendas específicas para os restaurantes, os agricultores e agroextrativistas mantêm seus canais de comercialização anteriores.

\section{Volumes adquiridos e oferta constante como fatores de instabilidade da gastronomia}

Funcionando como empreendimentos comerciais que atendem a uma lógica de mercado, os restaurantes sofrem com dilemas operacionais que se caracterizam por um discurso de sustentabilidade que não se reflete totalmente na prática. Nos estabelecimentos de maior 
porte/público ocorre o desafio de se conseguir comprar diretamente de produtores sem que haja uma nota fiscal. Outros desafios foram: inclusão de frutos do cerrado no cardápio permanente, tanto pela pouca flexibilidade de pratos sazonais, quanto pela própria aceitação dos consumidores que acabam por escolher pratos comerciais; falta tempo ou disposição do chef de ir atrás dos produtos, que demonstram a necessidade de uma cadeia de comercialização mais estruturada.

Para os dois estabelecimentos destacados, Buriti Zen e Mesa pra Doze, esses desafios aparecem em menor intensidade, pois a linha de gastronomia seguida por eles coloca em primeiro foco a questão ativista da causa socioambiental. Desse modo, prevalece para eles práticas associadas ao seu posicionamento político e ideológico. Desse modo os cardápios se adequam aos produtos disponíveis em cada época, respeitando a sazonalidade das espécies, priorizando a produção agroecológica disponível pelos produtores a eles mais vinculados.

\section{Novos mercados a partir da gastronomia}

Os desafios dos chefs de cozinha e restaurantes em manter pratos no cardápio que atendam a vários princípios de sustentabilidade na gastronomia refletem numa instabilidade na relação entre produtores e restaurantes. Além disso, por vezes o volume de produtos consumido pelos restaurantes, que possuem pratos com ingredientes da sociobiodiversidade, não chega a gerar uma grande renda para os produtores. Conforme relatado em entrevista com representante da cooperativa Central do Cerrado, os restaurantes com chefs renomados em Brasília acabam gerando pouco impacto financeiro direto, uma vez que seu consumo médio de pimenta de macaco (Piper aduncum), por exemplo, é de $1 \mathrm{~kg}$ a cada seis meses. Porém, quando esses chefs divulgam o uso desse ingrediente em entrevistas e em seus estabelecimentos para seus clientes, geram vendas indiretas, pois ocorre o aumento da procura dos mesmos ingredientes na cooperativa. Nessa situação os restaurantes agem como divulgadores dos produtos.

Apesar de os dois exemplos destacados nesse artigo, o Buriti Zen e o Mesa pra Doze, representarem um consumo maior de produtos da sociobiodiveridade, pois seus cardápios são sazonais e pensados a partir dos ingredientes, eles ainda entram nessa situação relatada pela cooperativa, pelo fato de atenderem um público pequeno. Em compensação, viabilizam a venda de produtos tanto das comunidades tradicionais quanto dos agricultores familiares do Entorno de Brasília e fomentam a aproximação de produtores com consumidores. O Buriti Zen abre seu espaço para ponto de encontro entre co-agricultores, venda de produtos da sociobiodiversidade e promove também debates e cursos sobre a alimentação tradicional com participação fundamental de lideranças das comunidades quilombolas Kalunga, como um ato de educar através da culinária.

Outro caminho interessante nesse processo de aproximação dos chefs com os produtores é a comercialização de produtos que geralmente não são vendidos nas feiras, como o caso da flor de abóbora relatado por Mateus Zanella do Mesa pra Doze:

A flor de abóbora é um caso muito específico aqui em Brasília, é uma delícia, mas os agricultores nem colhem porque não tem no mercado, eles não conseguem vender, então acaba apodrecendo no pé, geralmente. Então, a gente foi atrás de uma produtora do Ceasa, a Dona Fátima que a gente conhece e falou "Dona Fátima, você vende abóbora você consegue coletar a flor de abóbora pra mim?” Aí ela “Ah, beleza!”

Desse modo, além dos restaurantes proporcionarem a comercialização de produtos da sociobiodiversidade e da agricultura familiar para além do que é utilizado na elaboração do cardápio do restaurante, também fazem de seu espaço uma possibilidade de abertura de novos canais de comercialização, valorização dos produtores e resgate da cultura alimentar. 


\section{Diferença entre agricultores do Entorno de Brasília e comunidades tradicionais quilombolas e indígenas}

As comunidades tradicionais possuem uma relação diferente na comercialização dos seus produtos. Necessitam de um apoio de intermediação para abertura de canais para escoamento. No caso de Brasília, a rede de comercialização OCCA tem exercido importante papel nesse sentido. No geral as comunidades rurais sempre apresentam entraves em toda a cadeia produtiva e nos canais de comercialização dos produtos. Mas no caso das comunidades tradicionais isso fica mais evidente. Como relatado em entrevistas, seminários e em visitas a campo, a localização das comunidades muito distantes dos centros urbanos ou de pontos de comercialização faz com que o transporte seja apontado como a principal dificuldade para acessar os diferentes mercados. Em segundo lugar são apontadas as restrições para armazenamento, uma vez que nem todas as comunidades tradicionais possuem energia elétrica, o que impede o processamento e armazenamento de alguns produtos em condições adequadas, como para algumas frutas do cerrado, cajuzinho e mangaba (Harconia speciosa), que são muito perecíveis. Apesar de ter aumentado a disponibilidade de tecnologias para melhoria das etapas de pós-colheita (OLIVEIRA; SCARIOT, 2010; SAMPAIO; CARRAZZA, 2012), muitas delas não conseguem chegar a todas as regiões onde se pratica o extrativismo de espécies vegetais nativas, principalmente aquelas de difícil acesso ou com infraestrutura precária de serviços básicos, principalmente água encanada, energia e transporte (DINIZ, 2020).

Essa diferença também vai refletir no modo de relação comercial das comunidades tradicionais com os restaurantes. Com a dificuldade de transporte o agroextrativista precisa escoar o máximo da sua produção de uma só vez, como no caso da venda de cajuzinho do cerrado para o restaurante Buriti Zen. Enquanto isso os agricultores familiares próximos de Brasília têm condições de participar de feiras livres todas as semanas. A Feira da Agricultura Familiar promovida pela EMATER-DF e localizada na CEASA é um exemplo. É nessa feira que várias parcerias e contatos entre agricultores familiares e chefs de cozinha foram estabelecidos.

Outras iniciativas têm surgido nos últimos anos, além das feiras e CSAs, e que também se integram aos restaurantes do sistema alimentar alternativo. Mesmo com as dificuldades impostas pela pandemia de covid-19, produtores e chefs, algumas vezes com o apoio de atores institucionais ou até mesmo de grupos de consumidores, continuam se relacionando, na busca da garantia de uma alimentação sustentável para a população de Brasília.

\section{Considerações Finais}

Neste trabalho apresentamos experiências de inserção de produtos orgânicos, agroecológicos e da sociobiodiversidade do Cerrado em restaurantes de Brasília, que desencadeiam outras iniciativas relacionadas à gastronomia sustentável e que se inserem nos sistemas alimentares alternativos a partir do seu envolvimento com a alimentação contemporânea.

Parte da gastronomia em Brasília está voltada para práticas sustentáveis, como o uso de produtos locais e da sociobiodiversidade, porém, ainda é notória a fragilidade dessa relação, pois nem sempre os restaurantes, inseridos na lógica comercial, conseguem manter a periodicidade e o volume de compra suficiente para gerar um grande impacto positivo na renda dos agricultores e comunidades tradicionais. Apesar disso os restaurantes começam a somar funções como a de agregar outros serviços como educação alimentar, formação (oficinas), pontos de encontro de co-produtores, pontos de venda de produtos da agricultura familiar e da 
VALETTE, E.; SCHREIBER, K.; CONARÉ, D.; BONOMELLI, V.; BLAY-PALMER, A.; BRICAS, N.; SAUTIER, D.; LEPILLER, O. An emerging user-led participatory methodology. Mapping impact pathways of urban food system sustainability innovations. In: Blay-Palmer, A. et al. (Org.). Sustainable Food System Assessment: lessons from Global Practice. New York, Oxon: Routledge, 2019. pp. 19-41.

ZANELLA, M. A. On the challenges of making a sustainable kitchen: experimenting with sustainable food principles for restaurants. Research in Hospitality Management 2020, 10(1): 29-41 https://doi.org/10.1080/22243534.2020.1790207

ZANETI, T. B.; BALESTRO, M. V. Valoração de produtos tradicionais no circuito gastronômico: lições do Cerrado. Sustentabilidade em Debate. v. 6, n. 1, 2015, p.22-36.

ZANETI, T. B. (2017). Cozinha de raiz: as relações entre chefs, produtores e consumidores a partir do uso de produtos agroalimentares singulares na gastronomia contemporânea. Tese (Doutorado). Faculdade de Ciências Econômicas, Programa de Pós-graduação em Desenvolvimento Rural. Universidade Federal do Rio Grande do Sul, Porto Alegre.

ZANETI, T.; SCHNEIDER, S. (2017). O boom gastronômico como ferramenta para o desenvolvimento rural: uma análise do caso da Sociedad peruana de gastronomía - apega peru. In: COLLAÇO, J. H. L.; BARBOSA, F. A. C.; ROIM, T. P. B. Cidades e consumo alimentar: Tradição e modernidade do comer contemporâneo. Goiânia: Editora da Imprensa Universitária, 2017, p.53-79. 